\title{
RECONCILING THE NAVIGATIONAL GRID WITH THE \\ INERTIAL NAVIGATION SYSTEM
}

Lori D. Wanner

John Hopkins

Defense Mapping Agency Aerospace Center

\section{ABSTRACT}

The navigation grid overprinted on certain small scale navigation and planning charts published by the Defense Mapping Agency was originally intended for use in polar regions by navigators utilizing such techniques as celestial naviga$t i o n$ and LORAN-C. The use of grid north gave the navigator a uniform reference direction to use rather than the highly convergent meridians. The concept of a "non-changing north" is remarkably like that encountered in an inertial navigation system with the local-level platform untorqued (alpha-wander mode). However, the directional angles computed by the inertial navigation system rapidiy diverge from those measured from the charts. This paper explores the reason for this divergence and proposes a solution.

\section{INTROOUCTION}

The navigational grid is a rectangular network of straight lines overprinted on navigation and planning charts such that grid north coincides with the northward direction on the Greenwich Meridian. It provides a uniform pseudonorth as an azimuth reference rather than highly convergent meridians. The navigation grid is overprinted on several series of small scale (1:2,000,000 and smaller) charts, especially those to be used in the polar regions. However, one series, the Global Navigation Chart (GNC, scale $=1: 5,000,000$ ) series has the overprint on all charts in the series, even those near the equator. Our Jet Navigation Charts $11: 2,000,000$ and $1: 3,000,000)$ have the overprint on those charts covering the Continental United States for training purposes. The navigational grid was originally used for celestial navigation and radio aids such as LORAN-C and OMEGA.

Chart making presents the problem of representing the surface of a spheroid upon a plane surface. The surface of a sphere or spheroid is said to be undevelopable because no part of it can be flattened without distortion.

The desirable properties are:

1. True shape of physical features.
2. Correct angular relationship. A projection with this characteristic is said to be conformal or orthomorphic.

3. Equal area, or the representation of areas in their correct relative proportions.

4. Constant scale values for measuring distances.

lines.

5. Great circles represented as straight

6. Rhumb lines represented as straight lines.

It is possible to preserve any one and sometimes more than one property in any one projection, but it is impossible to preserve all of them. For instance, a projection cannot be both conformal and equal area, nor can both great circles and rhumb lines be represented as straight lines.

THE PROBLEM

Subpolar charts use the Lambert Conformal Conic projection. In this projection, the cone intersects the earth at two standard parallels. The area between the two standard parallels is compressed, and that beyond is expanded. If, in such a projection, the spacing of the parallels is altered so that the distortion is the same along them as along the meridians, the projection becomes conformal and is known as the Lambert Conformal Conic projection. It is the most widely used conic projection for navigation, though its use is more common among aviators than mariners. The modified Lambert Conformal Projection is virtually conformal over its entire extent, and the amount of its scale distortion is comparatively little if it is carried only to about $25^{\circ}$ or $30^{\circ}$ from the pole. Beyond this, the distortion increases rapidly. A great circle is very nearly a straight line anywhere on the chart. The Lambert Conformal Conic projection portrays longitide in an angle less than true longitude. When a flight crosses the $180^{\circ}$ meridian the Inertial Navigation System (INS) doesn't know it is supposed to "flip over," so the alpha angle, or angle between grid north and true north, just keeps increasing in magnitude instead of changing sign and decreasing. 
Polar charts use the Transverse Mercator Projection. A Transverse Mercator Projection is a conformal projection upon a plane. The cylinder is tangent along a meridian. Since the area of minimum distortion is near a meridian, this projection is useful for charts covering a large band of latitude and extending a relatively short distance on each side of the tangent meridian. This projection portrays meridians as slightly curved lines. Therefore, a great circle is actually a curved line on the charts. Alpha angle, measured on the chart, does not agree with the INS, nor does the yaw angle, the angle between grid north and the path frame measured clockwise, as measured on the chart.

These problems should be brought to the attention of the inertial navigation community so that the systems and programs which emulate systems can be modified.

\section{OBSERVATIONS}

We investigated 11 flight tracks selected to cover several conditions: hemisphere considerations, crossings of the $0^{\circ}$ and $180^{\circ}$ meridians, and polar and subpolar regions. Two projections were investigated; the subpolar Lambert Conformal Conic, with the standard paralle ls chosen to give the least deviation from true scale over the chart, and the Polar Transverse Mercator Projection with the true scale on $90^{\circ} \mathrm{E}$ and $90^{\circ} \mathrm{W}$.

The following formulas show how the Navigation Frame Heading Angle, Alpha, is computed. Alpha is measured counter clockwise from true north.

Lambert Conformal Conic Projection:

$\sin \phi_{0}=\left(\ln N_{1} \cos \phi_{1}-\ln N_{2} \cos \phi_{2}\right) /$

$\left[\ln \tan \left(45^{0}-\frac{1}{2} \phi_{1}\right)\left(\frac{1+e \sin \phi_{1}^{\frac{e}{2}}}{1-\operatorname{esin} \phi_{1}}\right)\right.$

$$
\left.\left.-\ln \tan \left(45^{0}-\frac{1}{2} \phi_{2}\right) \frac{1+e \sin \phi_{2}^{\frac{e}{2}}}{\left(1-\operatorname{esin} \phi_{2}\right.}\right)\right]
$$

where $\phi_{1}=$ upper standard parallel

$\phi_{2}=$ lower standard parallel

$\mathrm{e}=$ eccentricity of reference ellipsoid

$$
N_{j}=a /\left(1-e^{2} \sin ^{2} \phi_{j}\right)^{1 / 2}
$$

$\mathrm{a}=$ semimajor axis of reference ellipsoid

Then

$\alpha=-\lambda \sin \phi_{0}$ in the Northern Hemisphere (2)

$\alpha=\lambda \sin \phi_{0}$ in the Southern Hemisphere (3)

Transverse Mercator Projection:

$x=\frac{a}{2} \ln [(1+\cos \phi \sin \Delta \lambda) /(1-\cos \phi \sin \Delta \lambda)]$

$y=a \tan ^{-1}[\tan \phi \sec \Delta \lambda]$

where $\Delta \lambda=\lambda-\lambda_{0}$

$\lambda_{0}=\begin{aligned} & \text { longitude of central } \\ & \text { meridian of projection }\end{aligned}$

$$
\text { Then } \begin{aligned}
\frac{\partial x}{\partial \phi} & =\frac{1}{2} a\left[\frac{2 \sin \phi \sin \Delta \lambda}{1-\cos ^{2} \phi \sin ^{2} \Delta \lambda}\right] \\
\frac{\partial y}{\partial \phi} & =a\left[\frac{\sec ^{2} \phi \cos \Delta \lambda}{\cos ^{2} \Delta \lambda+\tan ^{2} \phi}\right] \\
\alpha & =\tan ^{-1}\left[\frac{\frac{\partial y}{\partial \phi}}{\frac{\partial x}{\partial \phi}}\right]
\end{aligned}
$$

if $\lambda>0$ in the northern hemisphere or if $\lambda<0$ in the southern hemisphere, then $\alpha$ equals the supplement of the angle computed in equation 8 .

On both the polar and subpolar charts, the yaw angle $\left(n_{z}\right)$ is computed by:

$$
\eta_{z}=\alpha+\psi
$$

where $\psi=$ angle between true north and path angle measured clockwise.

On the polar charts, computed angles and measured angles agree reasonably well. The al pha angle is very nearly equal in magnitude to the longitude of a point. The closer a flight track parallels grid north, the greater the agreement. The more a flight track is perpendicular to the grid north, and the farther it is away from the meridian of true scale, the lesser the agreement and the further a great circle deviates from a straight line.

On the subpolar charts, the problem is a bit more difficult. A difference in longitude is represented by an angle somewhat less than that difference. The ratio is known as the 
convergence factor and is given as a sin $\phi_{0}$, where $\phi_{0}$ is the latitude of a central parallel, which in turn is related to the two standard parallels of the projection. Equation (1), above, is the quite awesome formula for computing sin $\phi_{0}$, but conveniently it is given in the margin of the chart as the convergence factor. The product of this convergence factor and the longitudes is given in a table on the margin of the chart. (Figure 1)

\section{SOLUTIONS WE'VE TRIED}

One solution we tried on the polar charts was to compute a new grid using the Transverse Mercator Projection. The $0^{\circ}, 180^{\circ}, 90^{\circ} \mathrm{E}$, and $90^{\circ} \mathrm{W}$ lines were drawn at true scale. The great circles originating at these lines were plotted at an angle of $90^{\circ}$. Lines running North and South parallel to the meridian are straight lines, but the lines running East and west are curved lines. The only straight lines running East and West are the $90^{\circ} \mathrm{E}$ and the $90^{\circ} \mathrm{W}$. As one gets more and more away from the line of true scale, the lines deviate more and more.

If points on the surface of the earth are projected geometrically onto a tangent plane, from a point on the surface of the earth opposite the point of tangency, a stereographic projection results. The scale of the stereographic projection increases with distance from the point of tangency. An entire hemisphere can be shown on the stereographic projection without excessive distortion. Great circles through the point of tangency appear as straight lines. All other circles, including meridians and parallels, appear as circles or arcs of circles. We also tried plotting the new grid, with the $0^{\circ}$, $180^{\circ}, 90^{\circ} \mathrm{E}$, and $90^{\circ} \mathrm{W}$ lines drawn at true scale, on the stereographic projection. All lines except the $0^{\circ}, 180^{\circ}, 90^{\circ} \mathrm{E}$ and $90^{\circ} \mathrm{W}$ lines are curved lines.

\section{PROPOSED SOLUTIONS}

If a plane is tangent to the earth, and points are projected geometrically from the center of the earth, the result is a gnomonic projection. Distortion is so great that shapes, as well as distances and areas, are very poorly represented, except near the point of tangency. The usefulness of the projection rests upon the one feature that any great circle appears on the map as a straight line. This is apparent when it is realized that a great circle is the line of intersection of a sphere and a plane through the center of the sphere, this center being the origin of the projecting rays for the map. This plane intersects any other nonparallel plane, including the tangent plane, in a straight line. It is this one useful feature that gives charts made on this projection the common name greatcircle charts.
When we plotted the new grid on a gnomonic projection centered at the pole, we discovered that the measured angles are correct. To compute the alpha angle for the gnomonic projection in the polar region the following formulas are used.

$$
\begin{aligned}
& \text { Northern Hemisphere } \alpha=-\left(\lambda-\lambda_{0}\right) \\
& \text { Southern Hemisphere } \alpha=\left(\lambda-\lambda_{0}\right)
\end{aligned}
$$

We didn't find a solution for charts in the subpolar region. The alpha angle can be computed by using the convergence factor given on the margin of the chart and the following formu lae.

$$
\begin{aligned}
& \alpha_{c}=\lambda \sin \phi_{0}\left(\begin{array}{c}
\text { (with adjustment } \\
\text { hemisphere) }
\end{array}\right. \\
& \eta_{z t}=\eta_{z c t}\left(\alpha_{c}-\alpha_{t}\right)
\end{aligned}
$$

where $c=$ computed

$$
t=t i m e
$$

\section{CONCLUSIONS}

The navigation grid, which was of great use to pre-inertial navigators, can still be of great use. It may be necessary, however, to produce polar charts on a gnomonic projection and sacrifice the conformality inherent in the currently used projections. Since pilotage navigation has become less important a consideration, the sacrifice of conformality may not be as impalatible as it may seem. In the inertial navigation community it seems desirable to compute alpha from the formulae we have given. With the advent of strapdown systems with very sophisticated navigation computers, this seems to be a nominal task.

\section{REFERENCE}

Bowditch, N; American Practical Navigator; Defense Mapping Agency Hydrographic/Topographic Center, Pub. No. 9, Volume I; U.S. Government Printing office; 1984. 


\begin{tabular}{|c|c|c|c|c|c|c|c|c|c|c|c|}
\hline & & & & & & & & & & & \\
\hline 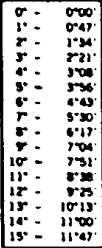 & 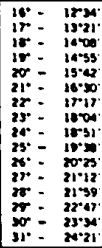 & 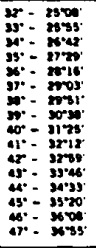 & 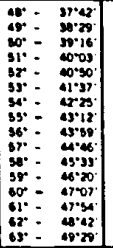 & 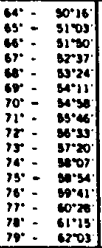 & 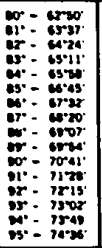 & 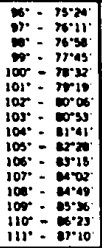 & 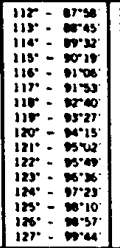 & 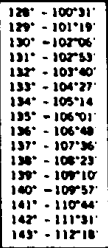 & 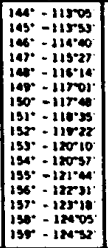 & 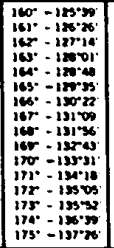 & 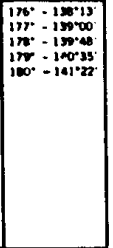 \\
\hline
\end{tabular}

\footnotetext{
Lomberl Conformal Conic Projection

Standord Poralle's $37^{\circ}$ and $65^{\circ}$

Scale $1: 5,000,000$

Convergence Forthr .78535
}

Figure 1. Convergence Table from Subpolar Chart 